\title{
Local eradication of rat colon cancer with photodynamic therapy: correlation of distribution of photosensitiser with biological effects in normal and tumour tissue
}

\author{
H Barr, P Chatlani, C J Tralau, A J MacRobert, P B Boulos, S G Bown
}

\begin{abstract}
Photodynamic therapy is a photochemical technique for the local destruction of tumours, entailing the interaction of light with an administered photosensitiser to produce a cytotoxic effect. We investigated the tissue distribution of the photosensitiser aluminium sulphonated phthalocyanine (AISPc) in dimethylhydrazine induced colonic tumours and adjacent normal colon in rats. Forty eight hours after intravenous injection, most tumours contained twice as much AlSPc as normal colon. Tumour size and position in the colon did not affect AlSPc concentration. Microscopic fluorescence localisation of AISPc showed significant photosensitiser accumulation in tumour stroma, whereas tumour and normal mucosa contained similar amounts. Thus, some normal tissue damage, where malignant cells invade normal areas, would inevitably accompany eradication of tumours. Tumour destruction and healing of colon after tumour eradication were examined histologically. There was sharp demarcation between necrotic areas (tumour or normal) and adjacent tissue and, whether the treated area was tumour or normal, healing occurred by regeneration of normal tissue. Some incompletely eradicated large tumours showed evidence of delayed bleeding. The possibility of selective uptake or preferential retention of the photosensitiser in tumours formed the initial basis for investigation of photodynamic therapy, but it is now clear that this is seldom the most important factor for tumour eradication. Of far greater importance is the nature of the biological effect of photodynamic therapy as necrosis of small tumours involving the full thickness of the bowel wall can be achieved with safe healing by regeneration of normal colon. The maximum depth of necrosis produced was only a few millimetres, so this technique is unlikely to be of value as the primary treatment for large colonic tumours but may prove of value for eradicating small lesions or as adjunctive therapy for eradication of small nests of tumour remaining or recurring in the tumour bed after conventional surgery.
\end{abstract}

Photodynamic therapy entails the administration of a photosensitiser with subsequent activation with light to produce a local cytotoxic effect. It initially attracted interest because parenterally administered photosensitisers, such as haemoporphyrin, showed increased fluorescence in malignant tumours in relation to surrounding normal tissue. ${ }^{1}$ Thus it seemed that an advantageous therapeutic ratio existed between normal and malignant tissue, although it is now clear that this tumour selectivity is not as dramatic as was first thought.

Cancer cells in general do not have higher affinity for porphyrin photosensitisers than normal cells. ${ }^{2}$ The early generalisation that haemoporphyrin derivative and dihaemoporphyrin ether/ester accumulate in malignant tissue to a higher degree than in normal tissue was disproved by distribution studies with radioactively labelled porphyrins. ${ }^{3}$ After intraperitoneal injection of $\left[{ }^{3} \mathrm{H}\right]-$ and $\left[{ }^{14} \mathrm{C}\right]-$ haemoporphyrin derivative in experimental animals most haemoporphyrin derivative was found in liver, spleen, and kidney. The best that could be achieved for a subcutaneous transplanted tumour was a tumour to skin ratio of 2:1. A more recent study has confirmed these findings and has shown that the skin adjacent to the implanted tumour has concentrations of dihaemoporphyrin ether/ester similar to that of the tumour, only muscle, brain, and distant skin accumulating less photosensitiser than the tumour. ${ }^{4}$ Although at present, haemoporphyrin derivative and its supposed active component, dihaemoporphyrin ether/ester, are the most widely studied photosensitisers, there is increasing interest in other groups of drugs, particularly the phthalocyanines, which have stronger absorption peaks further into the red part of the spectrum and little absorption elsewhere in the visible.

We have extensively investigated aluminium sulphonated phthalocyanine (AlSPc) as a photosensitiser for photodynamic therapy. Initial studies in chemically induced tumours in rodent colon and pancreas showed that there was a small therapeutic ratio (2:1) between the tumour and the adjacent normal tissue. ${ }^{6}$ By judicious manipulation of the treatment parameters, we have been able to exploit this to produce truly selective tumour necrosis - that is, necrosis in tumour but not in adjacent normal colon exposed to the same light dose - although only in small volumes of tumour. ' The ultimate goal, however, of selective tumour eradication, rather than just partial necrosis within a tumour mass, is more elusive. Studies using porphyrin photosensitisers for the eradication of tumours suggest that the normal tissue around the tumour must also be destroyed ${ }^{89}$ Nevertheless, there have been few studies to examine the effect of tumour eradication in the tissue of origin and the healing 
response of the surrounding normal tissue. Most experimental data has been gathered on transplanted tumours. Normal tissue damage may be acceptable if the tissue response is such that the healing occurs safely.

This paper presents further studies on the microscopic localisation of AISPc in experimental colonic tumours in relation to the adjacent normal colon to determine the distribution in the tumour and colon. These results are correlated with partial or total destruction of the tumours with photodynamic therapy and the subsequent healing.

\section{Methods}

PHOTOSENSITISER PREPARATION AND TISSUE ASSAY AlSPc was obtained from Ciba-Geigy and used dissolved in normal saline. ${ }^{5}$ The method of assay in tissue specimens has been described in detail previously. ${ }^{6}$ Briefly, after alkali extraction with $0.1 \mathrm{M}$ sodium hydroxide, the solution was centrifuged and the supernatant removed. The fluorescence from this was read on a spectrofluorimeter (Perkin-Elmer LS-5 Luminescence spectrofluorimeter) and AlSPc concentrations determined from a tissue corrected standard curve. The results were presented as $\mu \mathrm{g} \mathrm{AlSPc/g}$ tissue.

\section{COLON CANCER MODEL}

The animals used were 40 male Wistar weanling rats of approximately $100 \mathrm{~g}$ at the time of tumour induction with 1,2-dimethylhydrazine (DMH, Aldrich Chemical Co, Gillingham, Dorset). This was prepared by the method of Filipe ${ }^{10}$ and each rat received five subcutaneous injections of DMH ( $40 \mathrm{mg} / \mathrm{kg}$ body weight) into the left flank at weekly intervals. The animals were inspected weekly for illness with tumours developing at approximately 30 weeks. When any signs of illness developed or at regular intervals from this time on, the animals were anaesthetised (fentanyl and fluanisone) and the left colon inspected with a bronchoscope (Olympus BFlTR) to ascertain view for fiuorescence imaging using the CCD camera. whether tumours were present. A total of 78 tumours were produced.

\section{TISSUE DISTRIBUTION OF PHOTOSENSITISER}

\section{By chemical extraction}

Tumour bearing animals were photosensitised with a tail vein injection of $5 \mathrm{mg} / \mathrm{kg}$ AlSPc under general anaesthesia. They were allowed to recover and killed $3,24,48,72$, or 144 hours later. At postmortem, the entire colon was removed intact and opened on the antimesenteric border taking care to avoid incising a tumour. The mesentery was completely cleared from the bowel and all faeces thoroughly washed from the lumen. The predominantly polypoid tumours were carefully excised from the underlying and surrounding colon, if necessary, using an operating microscope (Wild M650). The portion of colon from which the tumour was removed was then excised and both specimens were placed in separate containers and frozen $\left(-20^{\circ} \mathrm{C}\right)$ until assayed. The uptake of AISPc was expressed as the ratio of the concentration in the tumour compared with that in the part of the colon in which that particular tumour arose. To examine the effect of tumour size on the uptake of photosensitiser, measurements of diameters were taken from any polypoid tumours that were nearly spherical (without ulceration or superficial necrosis) and the mean diameter calculated and correlated with AlSPc uptake. In two rats the tumours were multiple and evenly distributed throughout the entire colon. These animals were photosensitised and killed at 3 and 48 hours, and the tumours and adjacent colon collected and analysed as above but the tumour:normal ratio was correlated with the distance from the anus to identify any difference between tumours arising in different parts of the colon.

\section{By fluorescent microscopy}

We have previously described in detail the development, rationale, and technique for the microscopic localisation of AlSPc in tissue sections using fluorescence photometry. "This technique has proved invaluable for the study of the microscopic distribution of phthalocyanine photosensitisers. ${ }^{12}$ An inverted fluorescence and phase contrast microscope (Nikon Diapont) was coupled to a solid state CCD camera (Charge Coupled Device, Model 1, P8603, Wright Instruments) to record fluorescence images, or to a photomultiplier to measure fluorescence levels from specific spots in the tissue section. Figure 1 shows the apparatus with an excitation light source using a $1 \mathrm{~mW}$ helium neon laser $(632 \cdot 8$ $\mathrm{nm})$.

Tumour bearing animals were injected with AISPc $(5 \mathrm{mg} / \mathrm{kg}$ ) and killed from 1-168 hours later. Tumour and adjacent colon samples were taken and dropped into a beaker of $20 \mathrm{ml}$ of isopentane ( 2 methylbutane), which had been cooled in a Dewar flask of liquid nitrogen. The snap frozen tissue samples were stored in liquid nitrogen until sectioned. The tissue blocks were mounted on OCT medium (tissue tek II embedding compound, $\mathrm{BDH}$ ) and $6 \mu \mathrm{m}$ sections cut 
using a Cryocat E microtome (Reichert Ltd). The slides were maintained in liquid nitrogen until fluorescence microscopy was performed. The area to be examined was selected with phase contrast microscopy and the laser switched on and either the fluorescence emission from a single spot was measured with the photomultiplier or an entire fluorescence image of the section was obtained using the CCD camera and stored on the computer for later analysis. Data processing with the IBM PC allowed areas of interest in parts of the section to be analysed for quantitative analysis of the microscopic distribution. Fluorescence data from tumour stroma and mucosa, and adjacent normal mucosa, muscle, and serosa were obtained and the microscopic uptake in these areas compared at $1,12,48$, and 144 hours after photosensitisation. Since phase contrast images can be difficult to interpret, the position of the slide on the microscope stage was recorded. The slide was then stained with haematoxylin and eosin or elastic van Gieson (to identify collagen in the submucosa), the same area examined under conventional light microscopy, and a histological image stored and compared with the previously recorded fluorescence image, so allowing direct comparison. All quantitative data were corrected for autofluorescence from the background glasss slide and using tissue sections from unsensitised animals. Autofluorescence was very small and showed no difference between colon and tumour. For the data comparing readings taken from different parts of the same section, statistical analysis was performed using the Wilcoxon signed rank test.

\section{PHOTODYNAMIC THERAPY OF COLONIC TUMOURS}

The purpose of this part of the work was to eradicate individual tumours and observe the nature of the subsequent healing. Photodynamic therapy was performed 48 hours after photosensitisation (the time when the tumour:normal colon ratio was at its maximum, 2:1). The animals were anaesthetised and a midline laparotomy performed. The site of the tumour was identified by palpation and a longitudinal colotomy performed opposite the base of the tumour. All faeces were washed from the tumour and a light dose chosen (with reference to previous quantitative studies ${ }^{7}$ ) that would eradicate a

represents the ratio for an

individual tumour. The

horizontal lines are the mean ratio at each time.

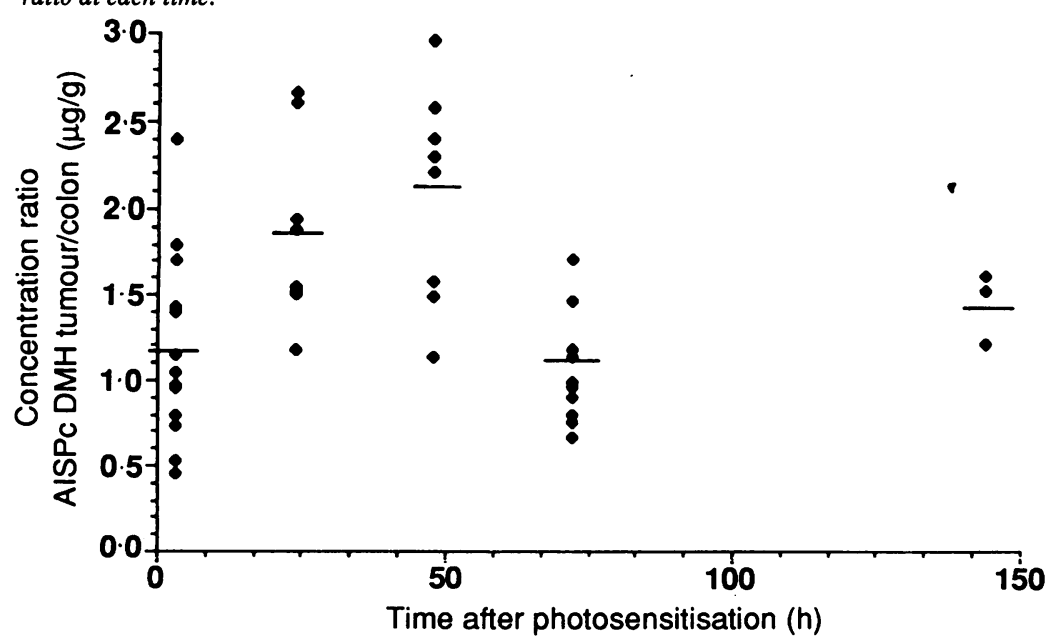

tumour of this size. Phototherapy was performed with an argon pumped dye laser (Aurora-Cooper Lasersonics) tuned to $675 \mathrm{~nm}$ and coupled to a $200 \mu \mathrm{m}$ quartz fibre. The laser power was set to $50 \mathrm{~mW}$, control studies having shown that this did not produce macroscopic thermal damage to these tumours after irradiation for 2000 seconds, although it must be assumed that there is a small area of thermal damage (typically $0.2 \mathrm{~mm}$ ) where the implanted fibre tip was in contact with the target tissue. ${ }^{5}$ The laser fibre was inserted at the apex of the tumour and the surrounding extracolonic organs shielded from inadvertent irradiation. After phototherapy the colotomy was closed transversely with interrupted $6 / 0$ silk sutures and the animal allowed to recover. The animals were killed 3, 24, 48, and 72 hours and 1 week, 2 weeks and 1 month after phototherapy. The section of colon where the treated tumour had been was removed, fixed in formalin, sectioned, and stained with haematoxylin and eosin for histological examination.

These tumours are prone to produce rectal bleeding and an attempt was made to clarify the risk of delayed haemorrhage that has been asssociated with photodynamic therapy. ${ }^{13}$ All animals were examined after phototherapy for evidence of rectal bleeding and at postmortem the entire colon was examined for blood. Evidence of bleeding was also sought in some animals with large tumours that were deliberately treated with a light dose that would only partially eradicate the tumour to see if this changed the risk of bleeding.

\section{Results}

AISPc UPTAKE IN TUMOURS

Figure 2 shows the ratio of AlSPc uptake between dimethylhydrazine tumours and adjacent normal colon as measured by chemical extraction. Each point is the ratio between the tumour and the colon in which it arose in the same animal. These data confirm our earlier study, ${ }^{6}$ although the maximum mean ratio 48 hours after photosensitisation is slightly smaller at just over $2: 1^{\dagger}$ compared with $2 \cdot 8: 1$. The striking feature is the range of values found with some tumours at 48 hours having the same concentration of AlSPc as the adjacent normal colon. Figure 3 shows the ratio correlated with the size of tumour and Figure 4 shows the ratio compared with the position in the colon. Neither of these two factors influences the therapeutic ratio.

MICROSCOPIC DISTRIBUTION OF AISPc

The table shows the quantitative results from fluorescence microscopy of tumour and adjacent normal colon. The most important finding is that the only significant difference between the highest fluorescence emission from any part of the tumour and from any part of the normal colon at the same time after sensitisation occurred 48 hours after photosensitisation and was between the normal mucosa and the tumour stroma. The malignant mucosa never contained significantly more AISPc than the adjacent normal mucosa. It 


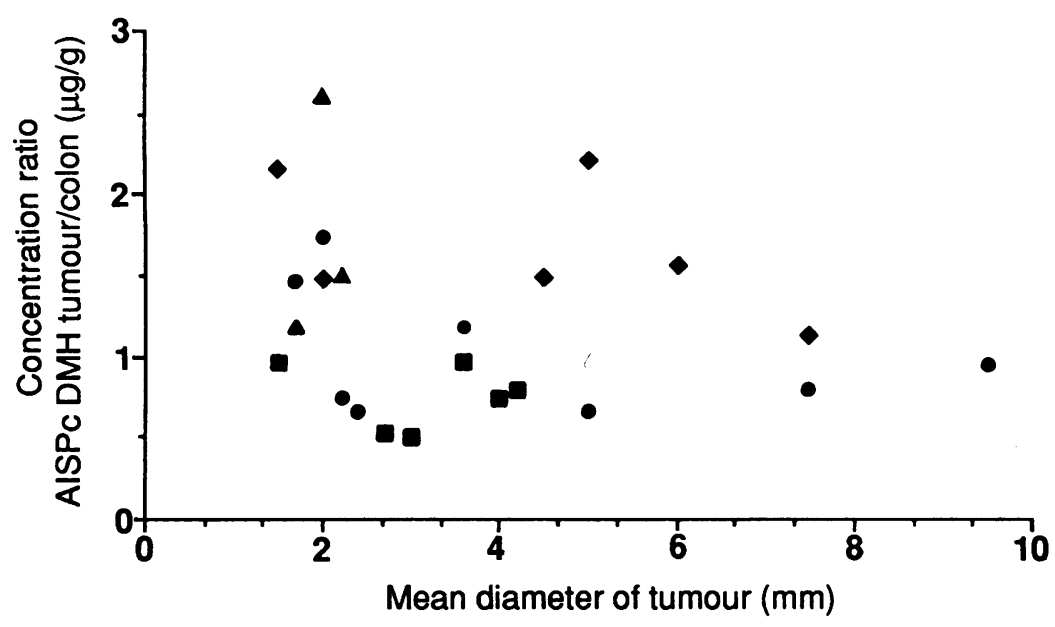

Figure 3: Ratio of concentration of aluminium sulphonated phthalocyanine $(A L S P C)$ in

dimethylhydrazine $(\mathrm{DMH})$ tumours to concentration in the adjacent normal colon in the same animal as a function of the mean diameter of the tumour after intravenous injection of 5 $m g / k g A l S P c, 3(\mathbf{\square}), 24$ $(\boldsymbol{\Delta}), 48(\bullet)$, and 72 hours (O) previously.

is also clear that the uptake of the photosensitiser is different in various parts of the tissue with the peak concentration occurring at different times. This phenomenon is being examined in more detail and is related to tissue vascularity and components that bind the photosensitiser.

\section{PHOTODYNAMIC THERAPY OF}

\section{DIMETHYLHYDRAZINE TUMOURS}

The histological progression of damage in normal colon after photodynamic therapy has been described previously. ${ }^{5}$ The nature of damage in tumours seen in this study was similar with an initial vascular dilatation, haemorrhage, and subsequent shutdown. Figure 5 shows a tumour 48 hours after photodynamic therapy. The necrotic tumour is separating from its pedicle and adjacent normal colon. The striking feature of this section is the sharp demarcation between necrotic and viable tissue, confirming the important fact that a threshold photodynamic therapy dose is required for necrosis in these tumours as in the normal colon. ${ }^{5}$ By 72 hours the tumour was necrotic and had separated and all that remained was a defect in a normal area of colon wall or a viable area of tumour.

Figure 6 shows an area of colon that previously contained a tumour. The tumour was treated with a photodynamic dose (photosensitiser/light combination) appropriate for complete eradication. At postmortem, there was no macroscopic abnormality but this area was found on serial section, showing regenerating mucosa encroaching on a small ulcerated area which was presumably the site of tumour attachment to the colon. Often it was not possible to identify the area where such tumours had been. In this photo-

Quantitative comparison of the fluorescence emission (arbitrary units) from dimethylhydrazine tumour and adjacent normal colon. Each mean (SEM) is from five to seven different specimens. All individual readings were from tumour and directly adjacent colon on the same section. Photosensitisation was with $5 \mathrm{mg} / \mathrm{kg}$ aluminium sulphonated phthalocyanine

\begin{tabular}{cllllll}
\hline $\begin{array}{l}\text { Time after } \\
\text { sensitisation } \\
\text { (hours) }\end{array}$ & Normal colon & & & \multicolumn{2}{l}{ Tumour } & \\
\cline { 2 - 4 } & Muscle & Serosa & Mucosa & & Stroma & Mucosa \\
\hline 1 & $87(4)$ & $302(45)$ & $292(67)$ & & $466(96)$ & $316(25)$ \\
12 & $54(10)$ & $223(45)$ & $330(74)$ & & $364(42)$ & $257(8)$ \\
48 & $60(21)$ & $68(28)$ & $87(24) \star \star$ & & $472(116)^{\star \star}$ & $46(8)$ \\
144 & $9(5)$ & $46(5)$ & $30(8)$ & & $28(9)$ & $13(4)$ \\
\hline
\end{tabular}

${ }^{\star \star} \mathrm{p}=0.023$ Wilcoxon signed rank test.

There was no other significant difference between the highest normal tissue fluorescence and the highest tumour fluorescence.

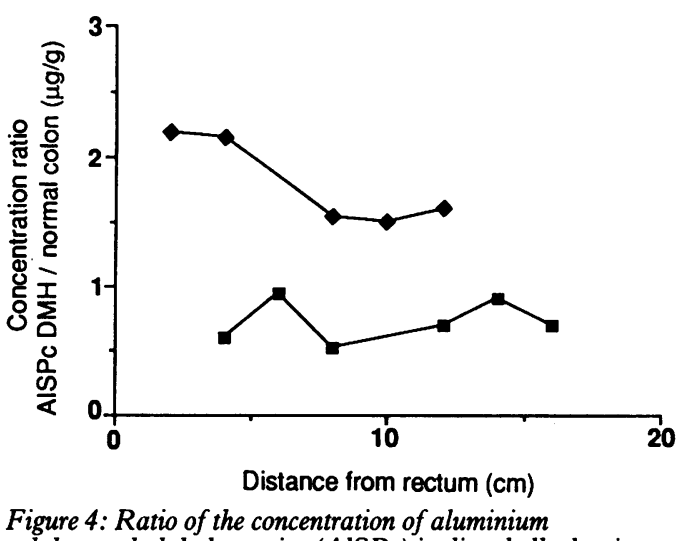

Figure 4: Ratio of the concentration of aluminium sulphonated phthalocyanine $(A L S P c)$ in dimethylhydrazine (DMH) tumours to concentration in adjacent normal colon of the same animal as a function of the position in the colon (distance from rectum) after intravenous injection of $5 \mathrm{mg} / \mathrm{kg}$ $A L S P, 3(\square)$ and 48 hours ( $)$ previously.

micrograph there is florid regeneration of mucosa with thickening of the submucosa and serosa. There is no muscle present under the small ulcer but the histological distribution of the muscle in relation to the tumour was not known before treatment. Thus no precise comment can be made.

There was no evidence of haemorrhage when tumour eradication was complete. In this series only three tumours showed evidence of haemorrhage. In none did the bleeding affect the clinical condition of the animal, and in all it was only discovered at postmortem. The feature of these tumours was that they had been substantially undertreated. They were relatively large (approximately $2 \mathrm{~cm}$ diameter), but after treatment a large area of viable tumour remained (diameter $1 \mathrm{~cm}$ ). Histology of these lesions did not show any exposed vessel that could have bled and was not remarkable in any other way. Six other undertreated large tumours showed no evidence of bleeding. The remainder of the tumours $(73 \%)$ were small $(<2 \mathrm{~cm})$ and $45 \%$ of these were totally eradicated.

\section{Discussion}

For the 'selective retention' or 'preferential uptake' of photosensitisers by malignant tissue to be clinically relevant there must be a therapeutic ratio between the tumour and the normal tissue in which it arose which can be exploited for therapy. It is surprising that so little work has been done on this aspect of photodynamic therapy. Many investigators only compare the uptake of the photosensitiser in subcutaneously transplanted tumours with that in adjacent muscle and skin and such results have little relevance to clinical practice for tumours of organs such as the colon. Recent data confirm that it is a misconception to state that photosensitisers are selectively retained by tumours compared with normal tissue. ${ }^{+}$Implanted subcutaneous tumours were found to retain dihaemoporphyrin ether/ester to a greater extent than the surrounding muscle but to retain less than the overlying skin and most of the other normal tissues examined. Our initial study of several tumours compared with the tissue of origin showed that the accumulation of the 


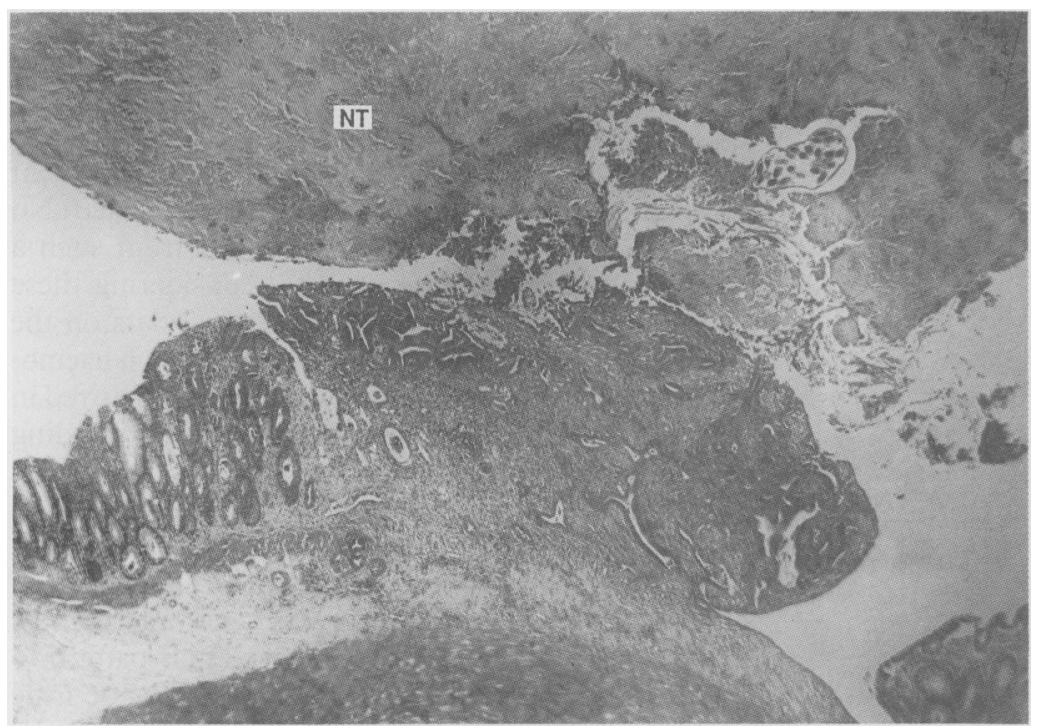

Figure 5: Necrotic tumour

$(N T)$ separating from the colon 48 hours after

photodynamic therapy.

Phototherapy 48 hours after intravenous injection of 5 $\mathrm{mg} / \mathrm{kg} A l S P c$, irradiation with $50 \mathrm{~mW}$ for 2000 seconds $(100 \mathcal{7})$

Haematoxylin E eosin, original magnification $\times 40$.

Figure 6: Area of colon, two weeks after photodynamic therapy, that previously contained a

dimethylhydrazine induced colonic tumour. The tumour has been totally eradicated. Phototherapy 48 hours after intravenous injection of 5 $\mathrm{mg} / \mathrm{kg}$ AlSPc, irradiation of the tumour with $50 \mathrm{~mW}$ for 2000 seconds $(100$ f). There was no macroscopic abnormality, but on serial section this area of regenerating mucosa with a small ulcer was seen. Haematoxylin E' eosin, original magnification $\times 40$. tumours were not allowed to develop further once the animals became symptomatic.

The localisation of AlSPc by fluorescence microscopy showed that only the stroma associated with the tumour contained the photosensitiser in significantly greater quantities than the adjacent normal tissue, even at the time of the peak therapeutic ratio, 48 hours after injection. Normal colonic mucosa and tumour mucosa contained similar amounts of AlSPc. These findings agree with those of Bugelski et $a l^{14}$ who investigated haemoporphyrin derivative distribution using autoradiography in implanted tumours. Our study establishes that AlSPc localises in a similar manner in an autochthonous tumour arising in its host tissue. Yet it was also shown that implanted polyvinyl sponges acting as model tumours localised porphyrin photosensitiser. ${ }^{15}$ These results suggest that the retention of the photosensitiser is a non-specific effect and have important implications for the investigation of photodynamic therapy.

To eradicate a tumour all malignant cells must be destroyed, in particular those in the area where the tumour is invading normal tissue. It is now apparent that there is no beneficial therapeutic ratio between normal and malignant cells in this region, so to eradicate a tumour it will be necessary to use treatment parameters that kill at least some normal tissue.

It is now clear that photodynamic therapy of implanted tumours with both porphyrin and phthalocyanine photosensitisers produces profound effects on the tumour microvasculature. ${ }^{816}$ Recent studies have shown that the overall tumour response is governed by vascular destruction and leads to increasingly nonselective tissue destruction as the photodynamic therapy dose increases. ${ }^{17}$ In particular, in order to eradicate a tumour using porphyrins as photosensitisers, normal tissue ${ }^{9}$ or normal vasculature ${ }^{8}$ has to be destroyed. It is clear that AlSPc uptake and microscopic distribution is very similar to that of porphyrins, and photosensitisers produce tissue damage in similar ways, so it can be expected that normal tissue would have to be destroyed to eradicate a tumour using the phthalocyanines as well. One aim of this study was to establish how the colon would heal after total destruction of the tumour. Healing occurred safely with regeneration of the colonic mucosa, so some degree of normal tissue damage is acceptable. Our previous studies showed that full thickness photodynamic therapy necrosis of normal rat colon healed without loss of mechanical strength as the submucosal collagen was undamaged and presumably acted as a template for healing by regeneration. ${ }^{18}$ For a tumour that involves the full thickness of the bowel wall the normal collagen of the submucosa will be destroyed, so the studies on normal bowel cannot be directly extrapolated to say that necrosis of these tumours will heal safely. The present experiments have, however, shown that even tumours reaching through to the serosa can be destroyed with safe healing. Histological sections of untreated full thickness tumours show abundant stromal collagen which would explain our experimental results. An example of this is shown in Figure 7. We have also looked at a small 


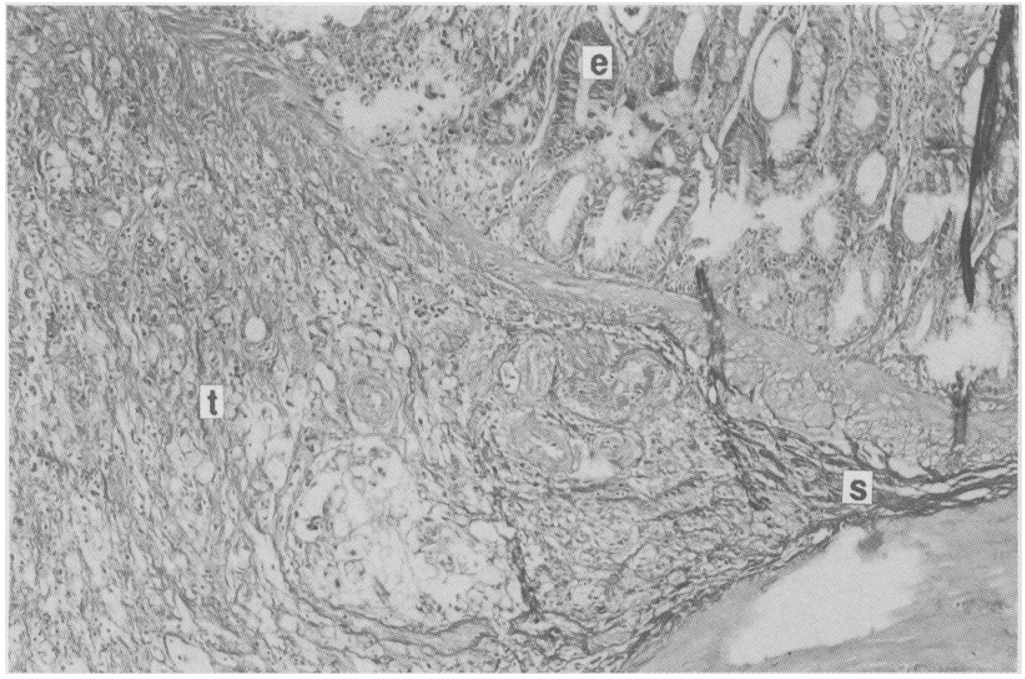

Figure 7:

Dimethylhydrazine induced colonic tumour. This shows the junction of normal and neoplastic tissue. The submucosal tumour mass $(t)$ is formed by tumour cells in stroma containing small collagen bundles. Epithelium (e) and submucosal collagen $(s)$ are also shown. Elastic van Gieson stain, original magnification $\times 100$, green filter.

Figure 8: Human colonic tumour. This shows a tumour acinus $(t)$ with thick adjacent collagen bands (c). Epithelium (e) and submucosal collagen (s) are also shown. Elastic van Gieson stain, original magnification $\times 100$, green filter. series of human colon cancers of the full thickness of the bowel wall after surgical removal (unpublished data) and shown that the stromal reaction includes production of large amounts of collagen (Fig 8), so it may be possible to use photodynamic therapy to treat small human colon cancers penetrating through to the serosa without risk of perforation.

In one small study of the treatment of skin nodules with photodynamic therapy a striking feature was the nature of healing. After treatment an area of skin necrosis occurred with eschar formation, but it caused minimal pain and healed without scarring. ${ }^{19}$ The nature of photodynamic therapy damage is different from thermal injury ${ }^{18}$ and radiation necrosis, which often fails to heal. ${ }^{1}$ These simple observations show important biological advantages for photodynamic therapy and provide a rational basis to proceed to clinical trials.

Reports of major haemorrhage occurring when necrosed tumour separated a few days after photodynamic therapy to advanced bronchial carcinomas $^{13}$ are of considerable concern, although it is remarkable how little has been done to study this potentially fatal complication. Unfortunately, it is difficult to explore this problem experimentally. We were hoping to clarify this in the present study. At postmortem three of the rat colon cancers had adherent blood clot after separation of the necrotic tumour after photodynamic therapy. It is striking that bleeding was never evident after photodynamic therapy to the normal colon ${ }^{5}$ nor was it evident when the tumour was completely eradicated. No definite conclusion can be drawn from such a small number of lesions, but considering these results with our own initial clinical data on the treatment of colorectal cancers ${ }^{20}$ in which haemodynamically significant haemorrhage occurred in one of 10 patients treated, it seems that bleeding is more prone to occur in large fleshy tumours which are only partly necrosed. The necrosed area seems to slough, leaving a raw tumour surface exposed that is likely to bleed.

In conclusion, the uptake and microscopic localisation of AISPc follow a similar pattern to that of other photosensitisers and provide little hope of truly selective tumour eradication. Photodynamic therapy with AlSPc, however, can be used to eradicate tumours in the rat colon by producing necrosis in the tumour and in the adjacent normal colon that heals safely by regeneration of normal tissues. The necrosis that can be produced in these tumours only extends a few millimetres from the light source ${ }^{7}$ and if large tumours are only partially eradicated there is a small risk of delayed haemorrhage, so photodynamic therapy is not likely to find a role as a primary treatment for tumours more than a few millimetres in depth. The results described here suggest that it may be useful as an adjunctive therapy. If the main bulk of tumour is removed by another technique such as surgery, photodynamic therapy may be capable of eradicating small foci of malignant cells in the tumour bed either at the time of the original procedure or in the event of subsequent local recurrence. As long as it is known that any necrosed normal tissues in the area will heal safely by regeneration after photodynamic therapy necrosis, which is the case for normal colon, it is safe to treat the entire tumour bed to give the maximum chance of eradicating all remaining nests of malignant cells.

In the early days of photodynamic therapy many unrealistic claims were made about its potential for selective destruction of malignant tumours. Now that its effects on normal and tumour tissue are better understood a more realistic picture is emerging. It is not suitable for the primary management of large tumours, but may have a useful clinical role in eradicating small tumours or as an adjunct to conventional management of larger lesions.

1 Gregorie HB, Horger EO, Ward JL, et al. Hematoporphyrinderivative fluorescence in malignant neoplasms. Ann Surg 1968; 167: 820-8.

2 Moan J. Porphyrin-sensitized photodynamic inactivation of cells: a review. Lasers Med Sci 1986: 1: 5-12

3 Gomer CJ, Dougherty TJ. Determination of $[3 \mathrm{H}]-$ and $[14 \mathrm{C}$ hematoporphyrin derivative distribution in malignant and normal tissue. Cancer Res 1979; 39: 146-51.

4 Bellnier DA, Ho Y-K, Pandey RK, Missert JR, Dougherty TJ. Distribution and elimination of Photofrin II in mice. Photochem Photobiol 1989; 50: 221-8.

5 Barr H, Tralau CJ, MacRobert AJ, et al. Photodynamic therapy in the normal rat colon with phthalocyanine sensitisation. BrF Cancer 1987; 56: 111-8.

6 Tralau CJ, Barr H, Sandeman DR, Barton T, Lewin MR, Bown SG. Aluminium sulfonated phthalocyanine distribution in rodent tumours of the colon, brain and pancreas. Photochem Photobiol 1987; 46: 777-81.

7 Barr H, Tralau CJ, Boulos PB, et al. Selective necrosis in dimethylhydrazine-induced rat colon tumours using phthalocyanine photodynamic therapy. Gastroenterolog 1990; 98: $1532-7$. 
8 Star WM, Marijnissen PA, van den Berg-Blok AE, Versteeg JAC, Franken KAP, Reinhold HS. Destruction of rat mammary tumor and normal tissue microcirculation by hematoporphyrin derivative photoradiation observed in vivo in Sandwich observation chambers. Cancer Res 1986; 46 in Sandwich

9 Henderson BW, Fingar VH. Relationship of tumor hypoxia and response to photodynamic treatment in an experimental and response to photodynamic treatment in
mouse tumor. Cancer Res $1987 ; 47: 3110-4$.

10 Filipe MI. Mucous secretion in rat colonic mucosa during carcinogenesis by dimethylhydrazine. A morphological and histochemical study. BrF Cancer 1975; 32: 60-77.

11 Barr H, Tralau CJ, MacRobert AJ, Morrison I, Phillips D, Bown SG. Fluorescence photometric techniques for determination of microscopic tissue distribution of phthalocyanine photosensitizers for photodynamic therapy. Lasers Med Sci 1988; 3: 81-6.

12 Chan W-S, MacRobert AJ, Phillips D, Hart IR. Use of charged coupled device camera for imaging of intracellular phthalocoupled device camera for imaging of intracellular

13 Cortese DA, Kinsey JH. Endoscopic management of lung cancer with hematoporphyrin derivative phototherapy. Mayo Clin Proc 1982; 57: 543-7.

14 Bugelski PJ, Porter CW, Dougherty TJ. Autoradiographic distribution of hematoporphyrin derivative in normal and tumor tissue of the mouse. Cancer Res 1981; 41: 4606-12. 15 Straight RC, Spikes JD. Preliminary studies with implanted polyvinyl sponges as a model for studying the role of neointertitial and neov neoilitis localisation, retor sensitizers. In: Kessel D, ed. Methods in porphyrin photosensitisation. New York: Plenum Press, 1985: 361-5.

16 Selman SH, Kreimer-Birnbaum M, Chauhhuri K, et al. Photodynamic treatment of transplantable bladder tumors in rodents after pretreatment with chloroaluminium tetrasulfophthalocyanine. F Urol 1986; 136: 141-5.

17 Henderson BW, Fingar VH. Oxygen limitation of direct tumor cell kill during photodynamic treatment of a murine tumor model. Photochem Photobiol 1989; 49: 299-304

18 Barr H, Tralau CJ, Boulos PB, MacRobert AJ, Tilly R, Bown SG. The contrasting mechanisms of colonic collagen damage between photodynamic therapy and thermal injury. Photochem Photobiol 1987; 46: 795-800.

19 Gilson D, Ash D, Driver I, Feather JW, Brown S. Therapeutic ratio of photodynamic therapy in the treatment of superficial ratio of photodynamic therapy in the treatment of superficial
tumours of skin and subcutaneous tissues in man. $B r \mathcal{F}$ tumours of skin and subc 1988 ; 58 : $665-7$.

20 Barr H, Krasner N, Boulos PB, Chatlani P, Bown SG. Photodynamic therapy for colorectal cancer: a quantitative pilot study. Br F Surg 1990; 77: 93-6. 